\title{
Impact of lymph node metastasis on outcome after extrapleural pneumonectomy for malignant pleural mesothelioma
}

Marc de Perrot, MD, Karl Uy, MD, Masaki Anraku, MD, Ming S. Tsao, MD, Gail Darling, MD, Thomas K. Waddell, MD, Andrew F. Pierre, MD, Andrea Bezjak, MD, Shaf Keshavjee, MD, and Michael R. Johnston, MD

Objectives: Extrapleural pneumonectomy is a therapeutic option for selected patients with malignant pleural mesothelioma. The impact of lymph node metastasis on the site of recurrence and the role of mediastinoscopy in the selection of patients for extrapleural pneumonectomy, however, remain unclear.

Methods: We reviewed 50 consecutive patients undergoing extrapleural pneumonectomy for malignant pleural mesothelioma in our institution between January 1993 and March 2005.

Results: The median survival was 11 months, with a 3-year survival of $24 \%$. Survival was significantly worse for patients with N2 disease than for those with no lymph node metastasis (median survival 10 months vs 29 months, respectively, $P=$ .005). Patient sex, histologic cell type, stage, and N2 disease, but not mediastinoscopy, had significant impacts on survival according to univariate analysis. In a multivariate analysis, however, only the presence of $\mathrm{N} 2$ disease remained a significant predictor of poor outcome. The proportion of patients with N2 disease and the long-term survival was similar regardless of whether preoperative mediastinoscopy yielded a negative result. The initial site of recurrence was determined in 28 patients (locoregional in 10 and distant in 18). The presence of N2 disease had no impact on the site of recurrence. Adjuvant hemithoracic radiation therapy, however, significantly decreased the risk of locoregional recurrence.

Conclusions: The presence of N2 disease negatively affects the prognosis of patients with malignant pleural mesothelioma. Mediastinoscopy, however, seems to have a limited role in patient selection for extrapleural pneumonectomy. Adjuvant hemithoracic radiation therapy but not $\mathrm{N} 2$ disease affects the risk of locoregional recurrence.

From the Division of Thoracic Surgery, Toronto General Hospital, University of Toronto, Canada.

Received for publication March 22, 2006; revisions received April 29, 2006; accepted for publication June 7, 2006.

Address for reprints: Marc de Perrot, MD, Division of Thoracic Surgery, Toronto General Hospital, 200 Elizabeth St, Toronto, M5G 2C4 Ontario (E-mail: marc. deperrot@uhn.on.ca).

J Thorac Cardiovasc Surg 2007;133:111-6 $0022-5223 / \$ 32.00$

Copyright $\odot 2007$ by The American Association for Thoracic Surgery

doi:10.1016/j.jtcvs.2006.06.044
$\mathrm{M}$ alignant pleural mesothelioma (MPM) is an uncommon neoplasm associated with poor prognosis. The median survival from the time of histologic diagnosis is approximately 12 months. ${ }^{1}$ The prognosis is worse for patients with extensive disease, poor performance status, or sarcomatoid histologic findings. ${ }^{2}$ Patients first seen with lymph node metastases have also been reported to have poorer prognosis. ${ }^{3}$ The impact of lymph node metastasis on the site of recurrence and the role of mediastinoscopy in the selection of patients for surgery, however, remain unclear. We therefore reviewed our experience of patients undergoing extrapleural pneumonectomy for MPM to assess the impact of lymph node metastasis on locoregional recurrence and the value of mediastinoscopy for patient selection.

\section{Patients and Methods}

A total of 50 consecutive patients with biopsy-proven MPM underwent extrapleural pneumonectomy in our institution between January 1993 and March 2005. Most patients (70\%, n 

Abbreviations and Acronyms
$\mathrm{CT}=$ computed tomography
MPM = malignant pleural mesothelioma

= 35) were operated on between June 2001 and March 2005. A retrospective chart review was performed after approval by the institutional review board.

All patients were evaluated preoperatively by chest radiograph, contrast-enhanced computed tomography (CT) of the chest and abdomen, spirometry, and ventilation-perfusion scan. Magnetic resonance imaging was used in selected cases. Brain CT or magnetic resonance imaging as well as bone scan were performed if clinically indicated. Resectability was defined by tumor confined to one hemithorax with no evidence of mediastinal organ, spine, diffuse chest wall involvement, or transdiaphragmatic extension. Positron-emission tomography was not available in our institution until recently and was not performed for any of these cases.

Mediastinoscopy was always performed if the mediastinal lymph nodes were at least $1.5 \mathrm{~cm}$ in their greatest diameter on the chest CT. If lymph nodes were smaller than $1.5 \mathrm{~cm}$ in their largest diameter, mediastinoscopy was performed on a case-by-case basis, mainly according to the surgeon's preference. Mediastinoscopy was performed in a standard fashion as previously described ${ }^{4}$ and was intended to systematically sample ipsilateral and contralateral lymph nodes of the upper mediastinum (upper paratracheal, lower paratracheal, and subcarinal stations). Patients with small $(<1.5$ $\mathrm{cm}$ in greatest diameter on chest CT) metastatic ipsilateral mediastinal lymph nodes found at mediastinoscopy were not necessarily excluded from surgery because these nodal stations were included in the postoperative hemithoracic radiation field.

Induction chemotherapy and adjuvant postoperative hemithoracic radiation have been systematically considered since June 2000 for patients undergoing extrapleural pneumonectomy for MPM in our institution. The protocol did not require systematic mediastinoscopy; if performed, however, the mediastinoscopy was usually done at the end of the chemotherapy, before the extrapleural pneumonectomy. Extrapleural pneumonectomy included en bloc resection of the lung, parietal pleura, ipsilateral diaphragm, and ipsilateral pericardium. Previous biopsy sites were removed, with a limited chest wall resection. Systematic mediastinal lymph node dissection or sampling was performed in all cases to allow accurate surgical staging of the disease. Paraesophageal, peridiaphragmatic, and subcarinal nodal stations were examined for tumors located on the right and on the left side. Additionally, paratracheal nodes were examined for right-sided tumors, whereas aortopulmonary window and para-aortic nodes were sampled for left-sided tumors.

Metastases to intrapulmonary, peribronchial, and hilar lymph nodes located within the pleural envelope were defined as N1 disease, whereas all metastases to ipsilateral lymph nodes located outside the pleural reflection were defined as N2 disease. Contralateral or supraclavicular lymph node metastasis was defined as N3 disease. The nomenclature used for non-small cell lung cancer and esophageal cancer was applied to define the location of extrapleural nodes. Tumors were staged according to the staging
TABLE 1. Characteristics of patients undergoing extrapleural pneumonectomy $(n=50)$

\begin{tabular}{lc}
\hline Age (y, median and range) & 58 (21-75) \\
Gender (male/female) & $42: 8$ \\
Tumor side (right/left) & $22: 28$ \\
Pathology (epithelial/other) & $36: 14$ \\
Mediastinoscopy (yes/no) & $29: 21$ \\
Induction chemotherapy (yes/no) & $34: 16$ \\
Adjuvant radiation therapy (yes/no) & $29: 21$
\end{tabular}

system developed by the International Mesothelioma Interest Group and published by the American Joint Committee on Cancer and the International Union Against Cancer. ${ }^{5}$

Postoperative deaths included all patients who died within 30 days of surgery or during the same hospitalization. Patients were followed up clinically and radiographically every 3 months with a physical examination and a chest radiograph or CT scan of the chest and upper abdomen. CT scans were always obtained if recurrence was suspected. Cytologic or histologic documentation of disease progression was performed if clinically indicated. Locoregional recurrence was defined as recurrence within the surgical or radiated field. Tumor progression outside the surgical or radiated field was considered to be distant recurrence. All patients were followed up until death or until June 2005.

Results are presented as absolute number and percentage or as median and range. Categorical variables were compared by $\chi^{2}$ analysis. Survivals were calculated from the time of extrapleural pneumonectomy by means of life-table analysis. Kaplan-Meier curves were plotted and compared by using the log-rank test for univariate analysis. Variables tested were patient sex (male vs female), mediastinoscopy (negative vs positive or not done), histologic type (epithelial vs other), induction chemotherapy (yes vs no), adjuvant radiation therapy (yes vs no), the presence of N2 disease (yes vs no), and stage (I/II vs III/IV). To avoid overadjustment by using too many variables in the multivariate models, only factors with a $P$ value less than .2 in the univariate analysis were considered in the multivariate analysis. Multivariate analysis was assessed by logistic regression analysis for categorical variables and by Cox proportional hazards stepwise model for numeric variables. StatView V (SAS Institute, Inc, Cary, NC) was used for all analyses.

\section{Results}

Patient characteristics are presented in Table 1. Most patients were male and had epithelial cell type. Four patients died postoperatively ( $8 \%$ ). The causes of death were bronchopleural fistula $(\mathrm{n}=1)$, cardiac arrhythmia $(\mathrm{n}=1)$, cardiac herniation after right pneumonectomy $(\mathrm{n}=1)$, and aspiration pneumonia $(\mathrm{n}=1)$.

A total of 29 patients had mediastinoscopy done before undergoing extrapleural pneumonectomy. The mediastinoscopy yielded negative results for metastatic lymph nodes in 26 patients. Three patients underwent extrapleural pneumonectomy despite positive results of mediastinoscopy for ipsilateral mediastinal lymph node metastasis. The medias- 
TABLE 2. Sites of extrapleural lymph nodes metastasis observed in 21 patients with $\mathbf{N} 2$ disease after extrapleural pneumonectomy

\begin{tabular}{lcc}
\hline Lymph node station* & $\begin{array}{c}\text { Right-sided tumor } \\
\text { (n = 11) }\end{array}$ & $\begin{array}{c}\text { Left-sided tumor } \\
\text { (n = 10) }\end{array}$ \\
\hline Upper paratracheal (station 2) & $1(9 \%)$ & - \\
Lower paratracheal (station 4) & $3(27 \%)$ & $4(40 \%)$ \\
Subaortic (station 5) & - & $4(40 \%)$ \\
Para-aortic (station 6) & - & $4(40 \%)$ \\
Subcarinal (station 7) & $10(91 \%)$ & $5(50 \%)$ \\
Anterior mediastinal & $3(27 \%)$ & $1(10 \%)$ \\
Internal mammary & $1(9 \%)$ & $1(10 \%)$ \\
Paraesophageal & $2(18 \%)$ & $4(40 \%)$ \\
\hline
\end{tabular}

*Most patients had more than one station involved.

tinoscopy was performed in the same operative setting as the extrapleural pneumonectomy in 21 cases (20 of these patients had induction chemotherapy) and within 6 weeks of extrapleural pneumonectomy in 6 cases. Two patients with negative results of mediastinoscopy underwent induction chemotherapy followed by extrapleural pneumonectomy 3 months later.

A total of 21 patients had no evidence of metastatic lymph nodes on final pathologic examination after extrapleural pneumonectomy, another 8 had N1 disease, and 21 had N2 disease. The locations of N2 lymph nodes according to the tumor side are summarized in Table 2. Patients with N2 disease had tumor in a median of 2 extrapleural lymph node stations (range 1-4). The subcarinal station was involved in 10 of 11 patients (91\%) with right-sided tumors and in 5 of 10 patients (50\%) with left-sided tumors. Eight patients had N2 disease and no intrapleural (N1) node metastasis.

Among 19 patients who had negative results of mediastinoscopy and underwent extrapleural pneumonectomy within the same operative setting, 9 had no metastatic lymph nodes found on final pathologic examination after extrapleural pneumonectomy (47\%), 4 had N1 disease (21\%), and 6 had N2 disease (32\%). In 2 of the patients with N2 disease, the positive extrapleural nodes were not reachable by mediastinoscopy, whereas in 4 the lymph nodes were sampled but no metastatic disease was detected on frozen section. Subcarinal node biopsies were falsely negative in 3 patients, and the left lower paratracheal lymph node was falsely negative in 1 patient with a left-sided tumor. The negative predictive value of mediastinoscopy for extrapleural metastatic lymph nodes was $68 \%$.

The overall median survival was 11 months, with a 3 -year survival of $24 \%$ (Figure 1). Survival was significantly worse for patients with N2 disease than for those with no lymph node metastasis (median survival of 10 months vs 29 months, respectively; $P=.005$ ). The median survival,

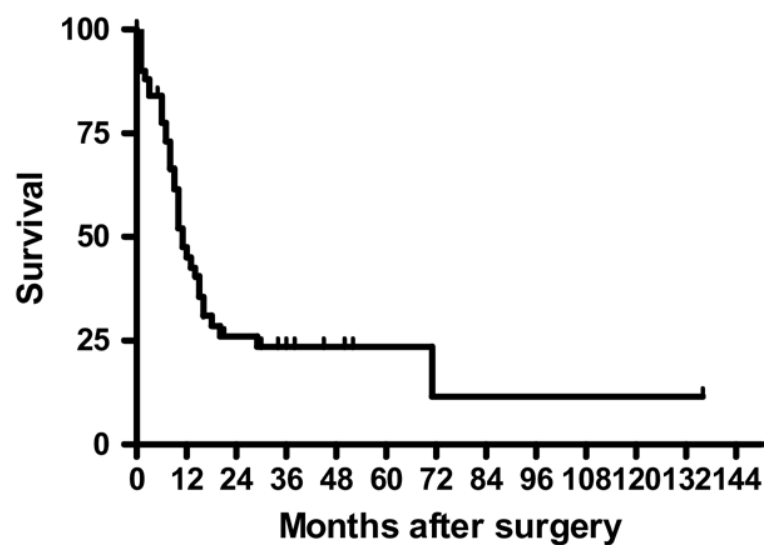

Figure 1. Overall survival of $\mathbf{5 0}$ patients undergoing extrapleural pneumonectomy.

however, was similar between patients with $\mathrm{N} 1$ and $\mathrm{N} 2$ disease (Figure 2). Among patients with N2 disease, the site of lymph node metastasis, the number of lymph node stations involved, or the presence of $\mathrm{N} 2$ disease with no intrapleural node involvement did not affect survival.

Univariate analysis demonstrated that sex, histologic type, N2 disease, and stage had a significant impact on survival (Table 3). Female patients and epithelial tumors were associated with better survival, whereas N2 disease and advanced stage were associated with worse survival. After multivariate analysis, however, only N2 disease remained a significant predictor of outcome. A negative result of preoperative mediastinoscopy had no significant impact on survival in either the univariate or the multivariate analysis.

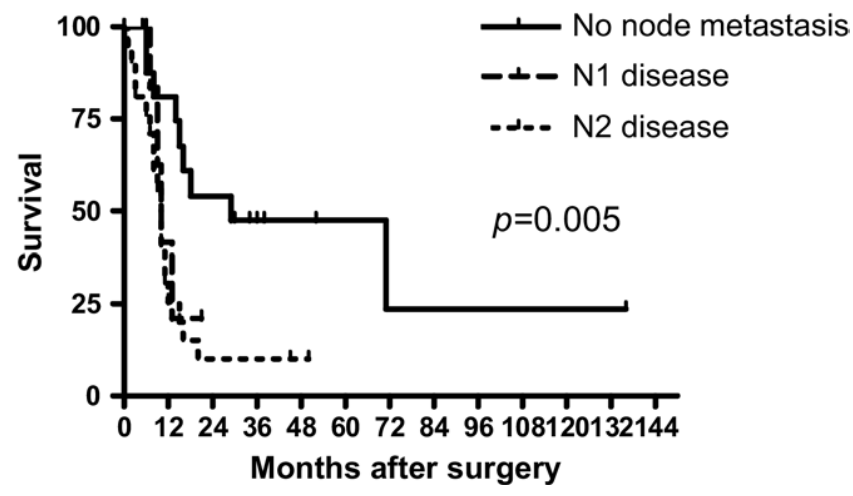

Figure 2. Survival of patients after extrapleural pneumonectomy according to presence of metastatic lymph nodes. Survival was significantly better for patients without metastatic lymph nodes than for patients with $N 1$ or $N 2$ disease $(P=.005)$. No difference in survival was observed between patients with N1 and N2 disease $(P=.5)$. 
TABLE 3. Impact of potential prognostic factors on longterm survival after extrapleural pneumonectomy by univariate and multivariate analysis $(n=46)^{*}$

\begin{tabular}{lcccc}
\hline & & $\begin{array}{c}\text { Univariate } \\
\text { analysis }\end{array}$ & \multicolumn{2}{c}{ Multivariate analysis } \\
\cline { 4 - 6 } Variable & $\mathbf{N}$ & $\boldsymbol{P}$ value & $\boldsymbol{P}$ value & $\begin{array}{c}\text { 95\% Confidence } \\
\text { interval }\end{array}$ \\
\hline Male patients & 39 & .008 & .09 & $0.8-19.1$ \\
Negative mediastinoscopy & 23 & .2 & .7 & $0.4-1.9$ \\
Epithelial cell type & 33 & .006 & .09 & $0.2-1.1$ \\
Induction chemotherapy & 30 & .9 & - & - \\
Adjuvant radiation therapy & 29 & .7 & - & - \\
N2 disease & 21 & .005 & .04 & $1.1-5.2$ \\
Stage III or IV & 38 & .03 & .8 & $0.3-3.9$
\end{tabular}

*Postoperative deaths $(n=4)$ were excluded from analysis.

The frequency of $\mathrm{N} 2$ disease diagnosed after extrapleural pneumonectomy was similar between patients who did not undergo preoperative mediastinoscopy and those who underwent extrapleural pneumonectomy after negative results of mediastinoscopy (Table 4). There was also no difference in the long-term survival between patients who had a negative mediastinoscopy result immediately before extrapleural pneumonectomy and those who had an extrapleural pneumonectomy without preoperative mediastinoscopy (Figure 3).

The initial site of recurrence was determined in 28 patients. Four patients had more than one site of recurrent disease at the time of relapse. Locoregional recurrence occurred in 10 patients, abdominal recurrence in 9, recurrence in the contralateral chest in 12 , and recurrence in the pericardium in 1. Locoregional recurrence was always located within the pleural space (even in patients with $\mathrm{N} 2$ disease) and was rarely associated with enlarging mediastinal nodes $(\mathrm{n}=1)$. Adjuvant hemithoracic radiation therapy was associated with a significantly lower risk of locoregional recurrence in both univariate and multivariate analyses (Table 5). Among patients with N2 disease, locoregional recurrence was detected in only 1 of 11 patients who underwent adjuvant hemithoracic radiation therapy, whereas it was observed in 5 of 9 patients who did not have

TABLE 4. Frequency of lymph node metastasis in patients undergoing extrapleural pneumonectomy with or without preoperative mediastinoscopy during the same operative setting

\begin{tabular}{lcc}
\hline $\begin{array}{l}\text { Postoperative nodal } \\
\text { staging }\end{array}$ & $\begin{array}{c}\text { Negative } \\
\text { mediastinoscopy } \\
(\mathbf{n}=\mathbf{1 9})\end{array}$ & $\begin{array}{c}\text { No } \\
\text { mediastinoscopy } \\
\text { (n = 21) }\end{array}$ \\
\hline No nodal disease & $9(47 \%)$ & $11(52 \%)$ \\
N1 disease & $4(21 \%)$ & $3(14 \%)$ \\
N2 disease & $6(32 \%)$ & $7(33 \%)$ \\
\hline
\end{tabular}

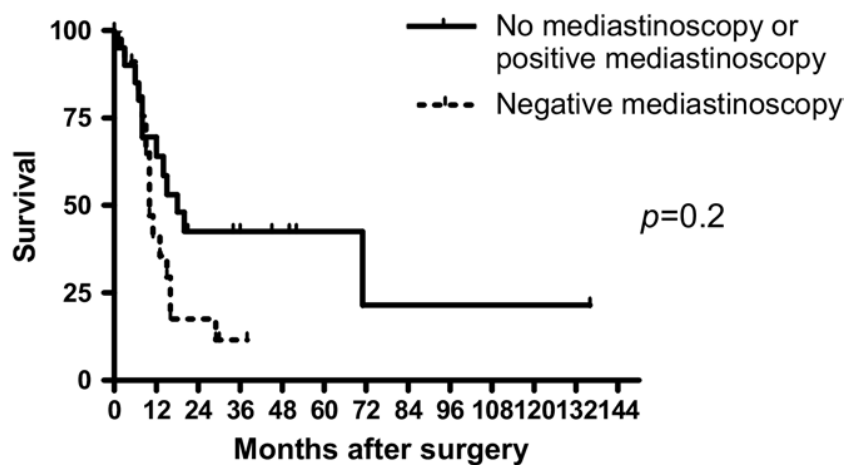

Figure 3. Survival was not significantly different between patients undergoing extrapleural pneumonectomy after negative results of mediastinoscopy and those undergoing extrapleural pneumonectomy without mediastinoscopy or after positive results of mediastinoscopy.

adjuvant radiation (9\% vs $56 \%$, respectively, $P=.02$ ). Better locoregional control was associated with a trend toward improved disease-free survival. Disease-free survival increased from 4.5 months for patients who did not undergo adjuvant hemithoracic radiation to 11 months when

TABLE 5. Impact of potential risk factors on locoregional and distant recurrence after extrapleural pneumonectomy

\begin{tabular}{|c|c|c|c|c|}
\hline \multirow[b]{2}{*}{ Risk factor } & \multicolumn{2}{|c|}{ Recurrence } & \multicolumn{2}{|c|}{$P$ value } \\
\hline & $\begin{array}{c}\text { Local } \\
(n=10)\end{array}$ & $\begin{array}{c}\text { Distant } \\
(\mathrm{n}=18)\end{array}$ & Univariate & Multivariate \\
\hline Sex & & & .2 & .9 \\
\hline Male & 9 & 18 & & \\
\hline Female & 1 & 0 & & \\
\hline Mediastinoscopy & & & .8 & - \\
\hline Negative & 5 & 10 & & \\
\hline Positive or not done & 5 & 8 & & \\
\hline Histologic type & & & .05 & .05 \\
\hline Epithelial & 4 & 14 & & \\
\hline Other & 6 & 4 & & \\
\hline Induction chemotherapy & & & .09 & .1 \\
\hline Yes & 4 & 13 & & \\
\hline No & 6 & 5 & & \\
\hline $\begin{array}{l}\text { Adjuvant radiation } \\
\text { therapy }\end{array}$ & & & .008 & .01 \\
\hline Yes & 2 & 13 & & \\
\hline No & 8 & 5 & & \\
\hline Nodal disease & & & .9 & - \\
\hline No or N1 & 4 & 7 & & \\
\hline N2 & 6 & 11 & & \\
\hline Stage & & & .6 & - \\
\hline | or || & 1 & 3 & & \\
\hline III or IV & 9 & 15 & & \\
\hline
\end{tabular}


hemithoracic radiation was administered after extrapleural pneumonectomy $(P=.1)$.

\section{Discussion}

This study supports previous publications demonstrating the negative impact of lymph node metastasis on outcome in patients with MPM. The 3-year survival reached $47 \%$ for patients without nodal metastasis, whereas it was only $10 \%$ for patients with $\mathrm{N} 2$ disease. There was no significant difference in median survival between patients with $\mathrm{N} 1$ and $\mathrm{N} 2$ disease. Although the number of patients is small, this finding supports the current TNM staging system published by the American Joint Committee on Cancer and International Union Against Cancer, which groups N1 and N2 node metastases as stage III disease.

The frequency in the literature of nodal metastasis in patients undergoing surgery for MPM varies between 25\% and $57 \% .^{3,6,7}$ Our experience is similar to that reported in the literature, with a frequency of approximately $50 \%$. The large majority of metastatic nodes in our series were located outside the pleural reflection. Few patients had intrapleural node metastasis only. As reported by other authors, subcarinal and paratracheal nodes are frequent sites of extrapleural metastasis. ${ }^{6,8}$ Metastasis to lymph nodes inaccessible by mediastinoscopy, however, such as the internal mammary chain, the anterior mediastinum, the aortopulmonary window, or the paraesophageal region, is also often seen. ${ }^{3,8}$

In our experience, the proportion of patients with metastatic lymph nodes after extrapleural pneumonectomy was similar regardless of whether the patients underwent preoperative mediastinoscopy. This finding can be explained by the rate of false-negative biopsy results from subcarinal and paratracheal nodes at the time of mediastinoscopy and by the presence of metastatic lymph nodes at stations inaccessible by mediastinoscopy.

The value of mediastinoscopy in patients with MPM was previously analyzed in two reports. ${ }^{9,10}$ Schouwink and colleagues ${ }^{9}$ observed that among 25 patients undergoing extrapleural pneumonectomy after negative mediastinoscopy results, 9 patients (36\%) were found to have N2 disease. ${ }^{9}$ In 6 patients the lymph nodes were not accessible by mediastinoscopy, and in 3 the low paratracheal and subcarinal lymph nodes were sampled but turned out to be positive only after the extrapleural pneumonectomy. ${ }^{9}$ Rice and associates ${ }^{10}$ reported that of 14 patients with positive ipsilateral mediastinal nodes after extrapleural pneumonectomy, only 5 were correctly identified preoperatively by mediastinoscopy. Thus, similar to our experience, approximately a third of the patients were found to have N2 disease after extrapleural pneumonectomy despite a negative result of mediastinoscopy.

Preoperative mediastinoscopy in patients who are otherwise candidates for extrapleural pneumonectomy can detect
N2 disease in about $15 \%$ of the patients and N3 disease in about 4\%. ${ }^{9,10}$ Mediastinoscopy detected 10 cases of N2 disease among 62 patients (16\%) evaluated for extrapleural pneumonectomy in the study reported by Rice and associates ${ }^{10}$ and 6 of $43(14 \%)$ in the study reported by Schouwink and colleagues. ${ }^{9}$ Rice and associates ${ }^{10}$ also found 4 patients with $\mathrm{N} 3$ disease at mediastinoscopy among 111 patients evaluated for extrapleural pneumonectomy (3.6\%).

The role of extrapleural pneumonectomy for patients with mediastinal lymph node metastasis is still controversial. ${ }^{6,9,10}$ Although the median survival after extrapleural pneumonectomy is only 10 to 12 months for patients with N2 disease, in our experience, and in that of others, ${ }^{9,11}$ some patients are still alive and disease free more than 3 years after surgery despite the presence of $\mathrm{N} 2$ disease. In addition, the introduction of postoperative hemithoracic radiation therapy can achieve excellent locoregional control even in patients with N2 disease. ${ }^{11}$

In our experience, approximately $35 \%$ of the patients had locoregional recurrence after extrapleural pneumonectomy. We observed, however, that adjuvant hemithoracic radiation therapy after extrapleural pneumonectomy significantly decreased the risk of locoregional recurrence, particularly when administered to patients with N2 disease. Fewer than $10 \%$ of the patients with $\mathrm{N} 2$ disease had locoregional recurrence if radiation therapy was administered postoperatively, whereas the rate was greater than $50 \%$ among patients with $\mathrm{N} 2$ disease who did not undergo adjuvant radiation.

There is no doubt that additional research is needed to improve our ability to adequately select candidates for extrapleural pneumonectomy. Standard cervical mediastinoscopy alone seems to have a limited role to improve this task; however, we acknowledge that our study is limited by the small total number of patients. The combination of mediastinoscopy with laparoscopy and possibly with contralateral thoracoscopy may increase the accuracy of staging. ${ }^{10,12}$ Aggressive mediastinoscopy with large sampling of the subcarinal nodes may also be important to improve the accuracy of mediastinoscopy, because station 7 is positive in a large proportion of patients with N2 disease. Positronemission tomography may have a role in patient selection in the future but seems more reliable to detect extrathoracic metastases than mediastinal nodal disease. ${ }^{13}$

In conclusion, the presence of $\mathrm{N} 2$ disease negatively affects the long-term survival of patients with MPM. Mediastinoscopy, however, seems to have a limited role in patient selection for extrapleural pneumonectomy. In addition, the administration of adjuvant hemithoracic radiation therapy appears to improve local control after extrapleural pneumonectomy, particularly for patients with N2 disease. Although there appears to be little indication currently for including mediastinoscopy in the treatment protocol of 
MPM, in the future, if stage-dependent treatment protocols are conceived, mediastinoscopy, in addition to other staging modalities, may become a more critical staging tool.

\section{References}

1. Robinson BW, Lake RA. Advances in malignant mesothelioma. N Engl J Med. 2005;353:1591-603.

2. Steele JP, Klabatsa A, Fennell DA, Pallaska A, Sheaff MT, Evans MT, et al. Prognostic factors in mesothelioma. Lung Cancer. 2005;49 Suppl $1:$ S49-52.

3. Sugarbaker DJ, Strauss GM, Lynch TJ, Richards W, Mentzer SJ, Lee $\mathrm{TH}$, et al. Node status has prognostic significance in the multimodality therapy of diffuse, malignant mesothelioma. J Clin Oncol. 1993;11: 1172-8.

4. Pearson FG. Mediastinoscopy: a method of biopsy in the superior mediastinum. J Thorac Cardiovasc Surg. 1965;49:11-21.

5. Rusch VW. A proposed new international TNM staging system for malignant pleural mesothelioma. From the International Mesothelioma Interest Group. Chest 1995;108:1122-8.

6. Pilling JE, Stewart DJ, Martin-Ucar AE, Muller S, Byrne KJ, Waller DA. The case for routine cervical mediastinoscopy prior to radical surgery for malignant pleural mesothelioma. Eur J Cardiothorac Surg. 2004;25:497-501.
7. Rusch VW, Venkatraman E. The importance of surgical staging in the treatment of malignant pleural mesothelioma. $J$ Thorac Cardiovasc Surg. 1996;111:815-26.

8. Rusch VW, Venkatraman E. Important prognostic factors in patients with malignant pleural mesothelioma, managed surgically. Ann Thorac Surg. 1999;68:1799-804.

9. Schouwink JH, Kool LS, Rutgers EJ, Zoetmulder FA, van Zandwijk $\mathrm{N}$, Vijver MJ, et al. The value of chest computer tomography and cervical mediastinoscopy in the preoperative assessment of patients with malignant pleural mesothelioma. Ann Thorac Surg. 2003;75: 1715-9.

10. Rice DC, Erasmus JJ, Stevens CW, Vaporciyan AA, Wu JS, Tsao AS, et al. Extended surgical staging for potentially resectable malignant pleural mesothelioma. Ann Thorac Surg. 2005;80:198893.

11. Rusch VW, Rosenzweig K, Venkatraman E, Leon L, Raben A, Harrison $\mathrm{L}$, et al. A phase II trial of surgical resection and adjuvant high-dose hemithoracic radiation for malignant pleural mesothelioma. J Thorac Cardiovasc Surg. 2001;122:788-95.

12. Alvarez JM, Ha T, Musk W, Robins P, Price R, Byrne MJ. Importance of mediastinoscopy, bilateral thoracoscopy, and laparoscopy in correct staging of malignant mesothelioma before extrapleural pneumonectomy. J Thorac Cardiovasc Surg. 2005;130:905-6.

13. Flores RM, Akhurst T, Gonen M, Larson SM, Rusch VW. Positron emission tomography defines metastatic disease but not locoregional disease in patients with malignant pleural mesothelioma. $J$ Thorac Cardiovasc Surg. 2003;126:11-6.

\section{The Journal of Thoracic and Cardiovascular Surgery Conflict of Interest Policy}

To assure fairness to authors submitting work for consideration in The Journal of Thoracic and Cardiovascular Surgery, a mechanism exists for managing conflicts of interest. The editor and each of the section editors complete a "Conflict of Interest" form that identifies any and all relationships with commercial and other academic entities. When the editor has a potential conflict because of a relationship with another entity or author, the editor appoints an alternate editor from among the section editors or editorial board members who assumes the entire responsibility for final decisions on the manuscript in question. The editor does not read the reviews that are submitted nor engage in discussing the manuscript prior to the final decision. When the conflict of interest involves a section editor, a "guest section editor" is appointed who fills the role normally played by the conflicted section editor. All members of the editorial board and reviewers are asked to indicate any conflict of interest when they agree to review a manuscript. 